\title{
Uveitis Specialists and Rheumatologists Select Different Therapies for Idiopathic Non-necrotizing Anterior Scleritis
}

\author{
Daniel J. Ozzello · Jason R. Kolfenbach · Alan G. Palestine
}

Received: September 11, 2016 / Published online: October 15, 2016

(C) The Author(s) 2016. This article is published with open access at Springerlink.com

\begin{abstract}
Introduction: Uveitis specialists and rheumatologists treat patients with anterior scleritis, but data from controlled trials to guide management are scarce, making differences in treatment paradigms possible.

Methods: 1044 uveitis specialists and rheumatologists were surveyed regarding therapy for a patient with anterior scleritis. Respondents were asked to select first- and second-choice therapies and then reselect therapies assuming that the costs of all options were equal and that insurance approval was ensured. Fisher's exact tests were employed to compare selections.
\end{abstract}

Results: Ninety-two respondents (8.6\%) completed the survey. Methotrexate was the

Enhanced content To view enhanced content for this article go to http://www.medengine.com/Redeem/ ED07F06040623D1D.

D. J. Ozzello · A. G. Palestine $(\bowtie)$

Department of Ophthalmology, University of

Colorado, Anschutz Medical Campus, Aurora, CO,

USA

e-mail: Alan.Palestine@UCDenver.edu

J. R. Kolfenbach

Division of Rheumatology, University of Colorado,

Anschutz Medical Campus, Aurora, CO, USA most-selected first-choice treatment before equalization of cost/insurance factors among uveitis specialists (44.4\%) and rheumatologists (78.6\%) $(p<0.009)$. Uveitis specialists selected mycophenolate at a higher rate $(27.8 \%)$ than did rheumatologists $(5.3 \%)(p<0.015)$. Cost and insurance considerations were not significant.

Conclusions: Uveitis specialists and rheumatologists have different preferences in the treatment of anterior scleritis. The difference is impacted more by specialty practice than by cost/insurance.

Keywords: Cost; Insurance; Scleritis; Specialty; Uveitis

\section{INTRODUCTION}

Anterior scleritis accounts for 99\% of cases of scleritis, the vast majority of which are due to a noninfectious etiology. The incidence of scleritis is approximately 3.4 per 100,000 person-years [1]. Scleritis is associated with a systemic inflammatory disease in $30-40 \%$ of patients $[1,2]$. In these patients, the treatment of ocular inflammation is partially dictated by 
treatment choices for the underlying systemic illness [3]. In patients without an underlying systemic illness, there is no clear consensus on optimal treatment regimen, and there are a wide array of potential therapeutic options [3-8].

Randomized controlled trials (RCTs) provide the highest level of evidence for treatment efficacy in the pharmacologic management of disease; however, scleritis has not been studied in this manner. There are several reasons for the lack of RCT data on this disease, including the cost considerations of carrying out such a trial and problems related to patient recruitment for a disease with low prevalence.

Formal treatment recommendations and guidelines are often derived from data from RCTs as well as meta-analyses; in their absence, recommendations are often formed by consensus of expert opinion. We have previously shown that subspecialists differ significantly in their recommendations for the management of intermediate and posterior uveitis [9-11]. These differences are influenced by subspecialty training as well as issues concerning cost and prior authorization by insurance companies. We sought to examine the treatment preferences of uveitis specialists and rheumatologists in the management of idiopathic scleritis, with the specific aim of identifying factors which influence treatment choice.

\section{METHODS}

We constructed an email questionnaire that included a brief case vignette describing a patient with idiopathic scleritis involving one eye. Exempt status was obtained from the Colorado Multiple Institutional Review Board; all data were collected voluntarily and anonymously. The survey was developed using Survey Monkey, and responses were collected through this online tool as well. Respondents to the survey were asked whether they were members of the American Uveitis Society, the American College of Rheumatology, or neither. Survey respondents were then presented with a clinical case vignette: "A 40 year old male has non-necrotizing painful nodular scleritis in the right eye. He has no systemic symptoms and laboratory work-up is negative. His scleritis has not been controlled with systemic NSAIDs and is still active on $20 \mathrm{mg}$ prednisone daily. He has been referred to rheumatology for consideration of systemic immunosuppression." Survey respondents were asked whether they would personally manage the patient or refer him to another specialist. Those who elected to manage the patient were asked to select first- and second-choice treatment options. The reason for needing to choose a second-choice option was not defined by the survey. They were then asked again for first and second treatment choices assuming that the cost to the patient was the same for any medication chosen and there would be no issues with prior authorization from the patient's insurance provider (cost/authorization equalized). Possible treatment choices were dexamethasone implant, fluocinolone acetonide implant, methotrexate, cyclosporine, mycophenolate mofetil, infliximab, adalimumab, rituximab, and "other" (a free text response that allowed respondents to enter additional treatment options not already specified). Periocular triamcinolone injections and azathioprine were frequent responses in this category and so were included in the analysis in their appropriate categories.

The survey was sent via email to members of the American Uveitis Society discussion group and to members of the American College of Rheumatology. Because the American College 
of Rheumatology is a much larger group, one-sixth of its members were randomly selected. Respondents to the survey from the American Uveitis Society discussion group were categorized as uveitis specialists. Those who responded from the American College of Rheumatology were categorized as rheumatologists. The Survey Monkey tool allowed the survey to be completed only once per computer, preventing multiple responses from a single recipient.

To analyze the effect of cost and prior authorization, treatment options were grouped into (1) local steroid injections (dexamethasone implant, fluocinolone acetonide implant, and periocular triamcinolone injection); nonbiologic immunosuppressive medications, also known as disease-modifying antirheumatic drugs (DMARDs: methotrexate, azathioprine, cyclosporine, and mycophenolate mofetil); (3) biologic immunosuppressive agents (infliximab, adalimumab, etanercept, and rituximab). Two-by-two contingency tables were used to compare the distribution of treatment choices before and after equalization of cost/ authorization. For each specialty, first choices were compared before and after cost and prior authorization were equalized, and then second choices were compared in the same manner using Fisher's exact test. Significant $p$ values were adjusted for false discovery rate/multiple comparisons where appropriate.

\section{RESULTS}

A total of 92 out of 1044 physicians completed the survey and were willing to recommend treatment for the scleritis case vignette (response rate 8.6\%). Thirty-six of the 192 email recipients $(18.8 \%)$ responded from the American Uveitis Society discussion group, while 56 of 852 rheumatologists (6.3\%) responded.

Table 1 shows the first-choice treatment preference for each specialty before and after equalization for cost and prior authorization. Methotrexate and mycophenolate mofetil were the most common agents chosen by both specialties. However, uveitis specialists and rheumatologists differed significantly in their usage both of methotrexate $(p<0.009)$ and mycophenolate $(p<0.015)$ before equalization of cost/insurance, with uveitis specialists choosing mycophenolate nearly as frequently as methotrexate and rheumatologists choosing methotrexate almost exclusively. These differences were not significant after equalization for cost/prior authorization when corrected for false discovery rate. Cost/ insurance authorization factors were not found to be significant for either uveitis specialists $(p=0.4561)$ or rheumatologists $(p=0.1714)$. There was an increased usage of biologic agents after adjustment for cost and prior authorization by uveitis specialists and by rheumatologists, but the change was only statistically significant for the rheumatologists $(p<0.05)$. Local steroid injection was the first choice of two rheumatologists (intraocular steroid implant) and five uveitis specialists (periocular triamcinolone).

Table 2 shows the second-choice treatment preference for each specialty before and after equalization for cost and prior authorization. Biologics were the most common agents chosen by both specialties, with $51.8 \%$ of rheumatologists and $27.7 \%$ of ophthalmologists preferring this approach (difference between specialties was not significant after correction for the false discovery rate). There was an increased usage of biologics after equalization for cost and prior authorization by uveitis specialists and by rheumatologists, but the increase was not 


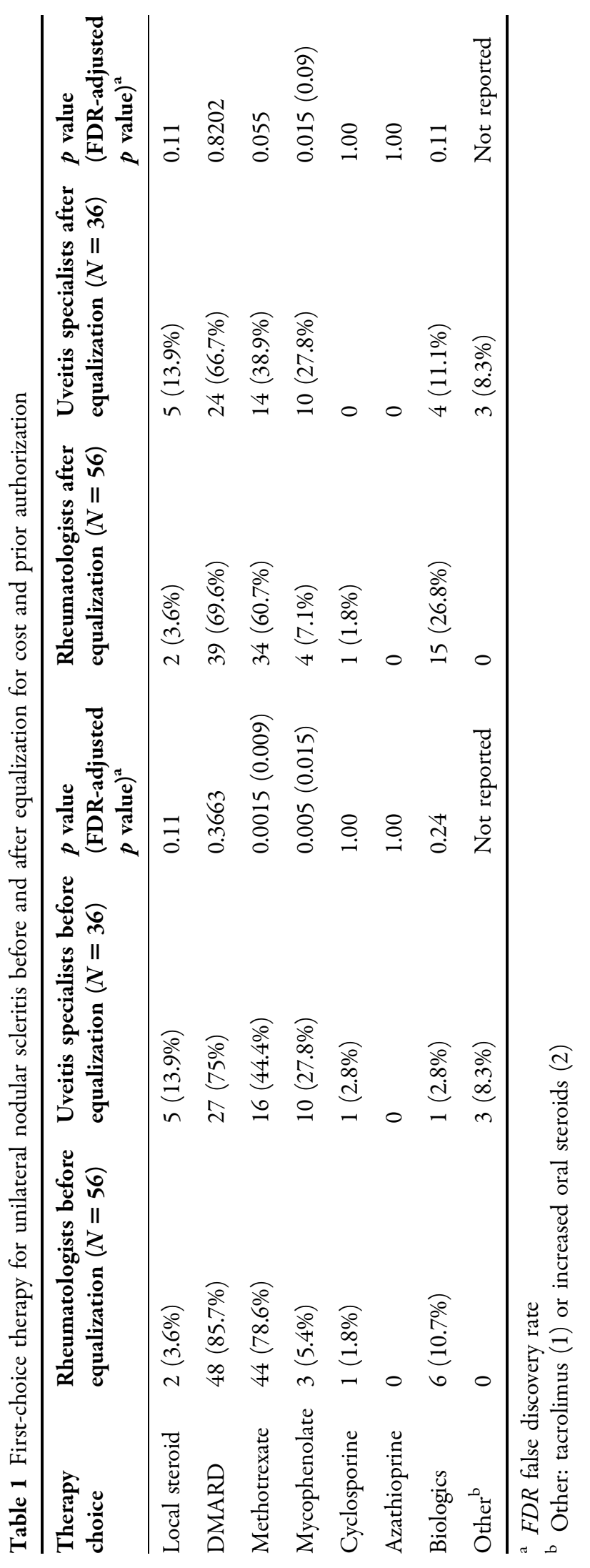




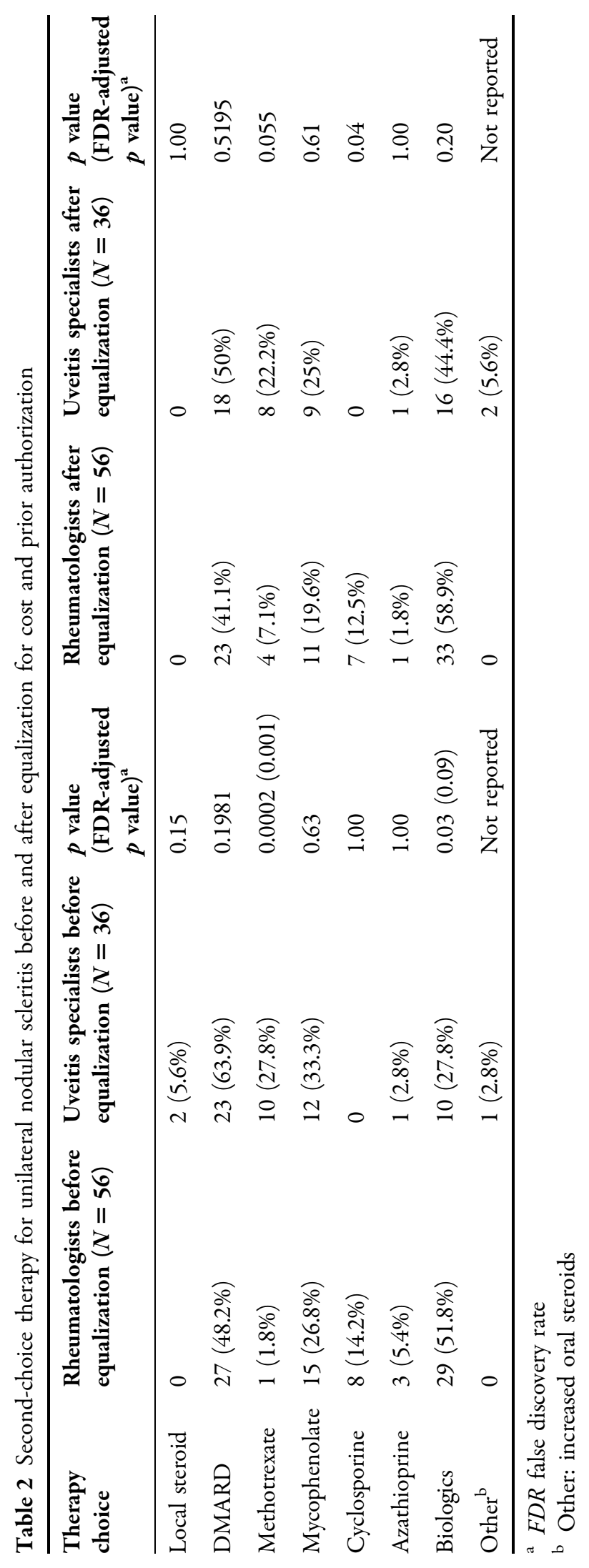


Table 3 Comparison of preference for methotrexate versus mycophenolate mofetil as first-choice therapy before and after equalization of cost/authorization among uveitis specialists and rheumatologists in the treatment of unilateral nodular anterior scleritis

\begin{tabular}{llll}
\hline & Uveitis specialists & Rheumatologists & Level of significance \\
\hline MTX/MM first choice (before equalization) & $16 / 10$ & $43 / 3$ & $p=0.0013$ \\
MTX/MM first choice (after equalization) & $14 / 10$ & $34 / 4$ & $p=0.0156$ \\
\hline
\end{tabular}

MTX methotrexate, $M M$ mycophenolate mofetil

statistically significant for either. Uveitis specialists and rheumatologists differed only in their usage of methotrexate $(p<0.001)$, and this difference did not persist after equalization of cost/insurance factors.

Anti-tumor necrosis factor (anti-TNF) agents and rituximab accounted for the biologic agents chosen. Specifically, $83.3 \%$ of the rheumatologists who chose a biologic as their first choice chose an anti-TNF agent, and 93\% chose an anti-TNF agent as their second choice. No uveitis specialists chose an anti-TNF agent as their first choice but $90 \%$ chose this as their second choice when selecting a biologic agent.

Table 3 focuses on the differing uses of the two most popular DMARDs, methotrexate and mycophenolate, as a first-choice therapy among rheumatologists and uveitis specialists. Rheumatologists significantly preferred methotrexate as a first-choice drug $(p<0.001)$. Methotrexate was also selected most often by uveitis specialists, but mycophenolate represented a significant minority of responses. This difference persisted after equalization for cost/prior authorization $(p<0.01)$.

\section{DISCUSSION}

Our data demonstrate that uveitis specialists and rheumatologists may have different preferences in the treatment of idiopathic nodular anterior scleritis. The lack of evidence-based medicine as well as differences in specialty paradigms for the treatment of scleritis lead to a wide spectrum of potential therapeutic choices within the standard of care. With the exception of corticosteroids, there are no Federal Drug Administration (FDA)-approved agents for the treatment of scleritis. Hence, all treatment options chosen by a clinician represent off-label use.

The majority of both types of specialists in our study chose a systemic immunosuppressive agent (DMARD) as their first therapeutic choice. However, there was a strong preference for methotrexate use by rheumatologists, whereas it was more likely for uveitis specialists to choose mycophenolate mofetil. This difference appeared to persist after equalization for cost and prior authorization, indicating that specialty treatment paradigms rather than cost may be an important determinant in therapeutic choices. Since the most common systemic disease associated with scleritis is rheumatoid arthritis, and methotrexate is a preferred treatment for this, we believe that this thinking is reflected in the higher use of this medication by rheumatologists. While both agents may be used to treat anterior scleritis that is inadequately controlled with oral NSAIDS and prednisone [6-8], this difference in usage has significant cost implications, as mycophenolate mofetil is approximately ten times more costly than methotrexate and has not been shown to be more efficacious $[9,12]$. 
Neither specialty frequently chose biologic agents as their first-choice therapy for this clinical vignette. This appears to be significantly different than we have demonstrated in ocular Behçet disease, where both specialties chose biologic agents as the most common first-choice intervention [10]. However, both the rheumatologists and the ophthalmologists in the current scleritis study most commonly chose biologic agents as their second-choice treatment, without a significant difference between specialties. It is interesting to note that the percentage of ophthalmologists who chose a biologic further increased after cost equalization, suggesting a greater sensitivity to treatment cost.

It is important to note that two rheumatologists and five ophthalmologists chose a local steroid injection as their first-choice treatment. The five ophthalmologists chose periocular steroid injections. Such injections have been shown to at least temporarily control symptoms and ocular inflammation, but there have been several case reports of extensive scleral melting after local corticosteroid injection in patients with scleritis, and this remains an area of controversy $[4,13,14]$. It is useful to understand that, in spite of this low risk, uveitis specialists may be willing to consider this option rather than using systemic immunosuppression.

A limitation of our study is that we presented only a single vignette for a patient with anterior scleritis, whereas there are often other factors that influence clinical decisions that we did not address. The low response rate of $8.6 \%$ of those surveyed could influence our results, since nonresponder bias may be due to survey recipients being less interested or familiar with treatment of scleritis. Furthermore, we were unable to track individuals who did not respond to our survey, which has the potential to lead to participation bias. In the United States, a large percentage of patients with scleritis are co-managed by rheumatologists and ophthalmologists who do not specialize in uveitis, and hence our results cannot be generalized to other countries.

Our study demonstrates that there may be significant differences in the therapeutic choices of specialists in the treatment of idiopathic scleritis. It is unlikely that direct comparisons among the therapeutic options for scleritis will be tested in a randomized controlled trial. All of the drugs preferred by both specialties have the potential for significant side effects and differ greatly in cost, without proof of superiority or even equivalence. In the absence of well-controlled studies, physicians must utilize information based on training, case series and reports, as well as personal experience and clinical judgment. Since these decisions have meaningful impacts on both healthcare cost and disease management, further investigation is warranted.

\section{CONCLUSIONS}

Methotrexate was the agent most commonly chosen by both specialties, but uveitis specialists were more likely to choose mycophenolate in the treatment of anterior scleritis. Cost and the need for prior authorization were not a significant factor in these differences, suggesting that subspecialty training plays a role in these treatment decisions.

\section{ACKNOWLEDGMENTS}

The authors received no funding support to conduct this study. All three authors were 
involved in the design of the study; data collection, analysis, and interpretation; and the preparation and review of the manuscript. The authors were solely responsible for the content and writing of the paper. All named authors meet the International Committee of Medical Journal Editors (ICMJE) criteria for authorship for this manuscript, take responsibility for the integrity of the work as a whole, and have given final approval for the version to be published.

Disclosures. D.J. Ozzello, A.G. Palestine, and J.R. Kolfenbach have nothing to disclose.

Compliance with Ethics Guidelines. Exempt status was obtained from the Colorado Multiple Institutional Review Board; all data were collected voluntarily and anonymously.

Open Access. This article is distributed under the terms of the Creative Commons Attribution-NonCommercial 4.0 International License (http://creativecommons.org/licenses/ by-nc/4.0/), which permits any noncommercial use, distribution, and reproduction in any medium, provided you give appropriate credit to the original author(s) and the source, provide a link to the Creative Commons license, and indicate if changes were made.

\section{REFERENCES}

1. Honik G, Wong IG, Gritz DC. Incidence and prevalence of episcleritis and scleritis in Northern California. Cornea. 2013;32(12):1562-6.

2. Lavric A, Gonzalez-Lopez JJ, Majumder PD, et al. Posterior scleritis: analysis of epidemiology, clinical factors, and risk of recurrence in a cohort of 114 patients. Ocul Immunol Inflamm. 2016;24(1):6-15.
3. Sainz de la Maza M, Molina N, Gonzalez-Gonzalez LA, Doctor PP, Tauber J, Foster CS. Scleritis therapy. Ophthalmology. 2012;119(1):51-58.

4. Watson PG. Treatment of scleritis and episcleritis. Trans Ophthal Soc UK. 1974;94:76-9.

5. Jabs DA, Mudun A, Dunn JP, Marsh MJ. Episcleritis and scleritis: clinical features and treatment results. Am J Ophthalmol. 2000;130(4):469-76.

6. Sen HN, Suhler EB, Al-Khatib SQ, Djalilian AR, Nussenblatt RB, Buggage RR. Mycophenolate mofetil for the treatment of scleritis. Ophthalmology. 2003;110(9):1750-5.

7. Thorne JE, Jabs DA, Qazi FA, Nguyen QD, Kempen JH, Dunn JP. Mycophenolate mofetil therapy for inflammatory eye disease. Ophthalmology. 2005;112(8):1472-7.

8. Jachens AW, Chu DS. Retrospective review of methotrexate therapy in the treatment of chronic, noninfectious, nonnecrotizing scleritis. Am J Ophthalmol. 2008;145(3):487-92.

9. Ozzello DJ, Palestine AG. Factors affecting therapeutic decisions in intermediate and posterior uveitis. Am J Ophthalmol. 2015;159(2):213-220.e213.

10. Palestine AG, Kolfenbach JR, Ozzello DJ. Rheumatologists and ophthalmologists differ in treatment decisions for ocular Behcet disease. J Clin Rheumatol. 2016;22(6):316-9.

11. Ozzello DJ, Singh J, Kolfenbach JR, Palestine AG. Specialty practice and cost considerations in the management of juvenile idiopathic arthritis-associated uveitis. J Pediatr Ophthalmol Strabismus. 2016;53(4):246-51.

12. Rathinam SR, Babu M, Thundikandy R, et al. A randomized clinical trial comparing methotrexate and mycophenolate mofetil for noninfectious uveitis. Ophthalmology. 2014;121(10):1863-70.

13. Jabs DA. Subconjunctival corticosteroids should not be used in the treatment of scleritis (discussion). Ophthalmology. 2002;109:806-7.

14. Johnson KS, Chu DS. Evaluation of sub-Tenon triamcinolone acetonide injections in the treatment of scleritis. Am J Ophthalmol. 2010;149(1):77-81. 